Grant DE-FG05-88ER13859

REPORT

November 2009

\title{
SEPARATION OF HIGHLY COMPLEX MIXTURES BY TWO-DIMENSION LIQUID CHROMATOGRAPHY
}

\author{
Principal Investigator \\ Georges Guiochon \\ Department of Chemistry, The University of Tennessee
}

\begin{abstract}
This report summarizes the progress made on the title project during the grant period. We developed a new classification of two-dimensional separations based on the observation that separations can be made in time or in space. Thus, two-dimensional separations can be made in timextime, spacexspace, spacextime, or timexspace. The two successive separations must use two different modes of chromatography that afford uncorrelated or weakly correlated patterns of retention factors for the components of the samples analyzed. Our attention was mainly focused on the separation of protein digests, particularly, on those of the digests of myoglobin and bovine serum albumin as model systems and extremely efficient temporal separations were developed. We also designed and constructed new instruments to carry out spacexspace separations (True Bidimensional Chromatography, $\mathrm{HPLC}^{2}$ or spacial separations) and timexspace separations (a new hybrid combination of a temporal and a spacial separation that we designed).
\end{abstract}




\section{INTRODUCTION}

A brief review of the aim of the proposal provides a perspective for the work that was carried out. The theoretical and experimental work performed, our results, and the present status of the project are described and the research plan for the end of the period is given.

\section{A - SCIENTIFIC OBJECTIVES OF THE ORIGINAL PROPOSAL}

The objective of the proposal was the investigation of the various possibilities available to separate a complex sample by combining two successive conventional separations by high performance liquid chromatography (HPLC) in order to better resolve the sample components by separating on the second system the compounds coeluting from the first one. Two widely different separation mechanisms are then needed to resolve the components of a complex sample that cannot be separated in a single step.

\section{AIM of the RESEARCH.}

The development of modern materials using complex polymer mixtures, the increasing concerns for the safety of our environment and the healthy character of our foods, our improved understanding of the molecular interactions that take place in biological systems are posing new series of difficult analytical problems. Forty years ago, relevant analytical problems could be solved using either a separation or a spectroscopic method and the choice of the best method depended mostly on the skills of the analyst and the tools available to him. Later, we learned to identify the most suitable method to solve a given type of analytical problems. About twenty years ago, it became clear that new, difficult problems could be solved only by coupling a separation and a spectroscopic method. Successively, GC-IR, GC-MS, LC-MS, and, more recently, LC-NMR became widely used in application laboratories. It is clear now that simple hyphenated methods cannot solve a variety of complex analytical problems. We must develop more complex separation schemes than those permitted by the current implementations of classical separation methods. This is why considerable attention is devoted to the direct coupling of two separation methods and justifies the interest that multidimensional HPLC is attracting. The aim of our research was to investigate the various possibilities available in this area.

\section{PROPOSAL OBJECTIVES.}

The purpose of our research was a fundamental investigation of the various methods available for the coupling of two separate chromatographic separations that would considerably enhance the resolution of the components of complex mixtures. This gain arises from (1) the combination of two independent retention mechanisms, one of them permitting the separation of the components that coelute in a phase system using the other mechanism, and (2) an enlargement of the separation space, allowing the separation of 
many more compounds in a given time. Many modes of separation are implemented in HPLC, using different kinds of molecular interactions, e.g., hydrophobic or hydrophilic interactions, polar interactions, hydrogen bonding, complex formation, ionic interactions, steric exclusion. This offers the wide variety of possible combinations discussed below.

Our main objectives were reached. We showed that the proper combination of two time separations performed on classical HPLC columns offers advantages (a larger separation power) and inconvenients (a longer analysis time) between which analysts must compromise [1]. We investigated the different possible combinations of space and time separations and showed that combining such separations offers a different balance of inconvenients and advantages (among which a much better detection sensitivity due to a decreased dilution of the sample components during their separation) than conventional combinations of two time separations [1]. We developed a new combination of column separations that yielded an extremely high separation power (characterized by a peak capacity of 7000) of the trypsin digest of bovine serum albumin and optimized the experimental conditions for achieving a given peak capacity in the minimum analysis time. Finally, we built an instrument implementing spacexspace separations, successfully packed its column, and selected the best retention mechanisms. We designed and are building a detector having the performance required to prove the validity of the method.

\section{B - REPORT OF TECHNICAL PROGRESS}

Our work involved three different issues: (1) a classification of the various possible implementations of two-dimensional HPLC [1]; (2) the development of a procedure to achieve a very high resolution power for a peptide mixture, using an off-line combination of two column separations [2]; and (3) the construction and operation of a simple spacexspace instrument for two-dimensional chromatography [3].

\section{FUNDAMENTALS Of BIDIMENSIONAL CHROMATOGRAPHY}

The separation power of HPLC is limited by the great difficulties encountered in preparing highly efficient columns and using them to separate the components of complex mixtures [4]. Only multi-dimensional HPLC could allow significant improvement of this situation.

\section{Column Efficiency and Peak Capacity.}

The separation power of HPLC columns is often characterized by its efficiency or number of theoretical plates $[1,4-7]$. The important parameter in the study of complex separations, however, is the actual number of peaks that the column can separate in a certain time. The peak capacity is the total number of peaks that can be lined up with a resolution unity, within a given time interval. It is simply related to the column efficiency, to the retention window or distance between the first and last peak considered, and to the resolution required between the peaks [5-7]. Different equations apply in isocratic and gradient elution 
chromatography. In practice, under optimum conditions, the peak capacity of a column having an efficiency $N$ is approximately $\sqrt{ } N / 2$ at a resolution unity in isocratic elution and slightly larger than $2-3 \times \sqrt{ } \mathrm{N}$ in gradient elution. The best unidimensional separations published so far correspond to values between 1,000 and 1,200.

In principle, the peak capacity of a bidimensional separation scheme is equal to the product of the peak capacities in each direction [1]. In practice, however, it is not possible to couple two separations made in different dimensions without some loss in separation power due to back-mixing during the transfer of the fractions from one dimension to the other and to a degree of correlation between the two retention mechanisms used.

\section{2 - Statistics of Band Overlap.}

In practice, the retention factors of the components of complex mixtures are randomly distributed, following approximately a Poisson distribution [8-10], a result extended to the distribution of the concentrations of the mixture components [11-13]. Thus, it is difficult to separate mixtures having a number of components that is comparable to the peak capacity. A statistics of band overlap was developed to relate the probable number of distinct peaks or bands observed when a $m$-component mixture is analyzed on a separation system that has a $n_{c}$-peak capacity [7-13]. This statistics affords a simple equation that gives the probability $P_{m}$ that a chromatographic system using $\mathrm{n}$ separation dimensions separates completely an $m$-component mixture, giving $m$ bands with a resolution unity, on an $n$ dimension column with a total capacity $n_{c}[13]$ :

$$
P_{m}=e^{-\phi_{n} 2^{n-1} \frac{m^{2}}{n_{c}}}
$$

where $\phi_{n}$ is a geometrical coefficient that accounts for the volume lost between spherical bands resolved in an $n$-dimension space. If we assume a compact $\mathrm{nD}$ lattice of spherical bands, we have for values of $\phi_{n}: 1,0.91$, and 0.74 for $n=1,2$, and 3 , respectively. For $n$ $=1$, the equation becomes $P_{m}=\exp \left(-m^{2} / n_{c}\right)$. For the lack of experimental data, few new advances were recently made. Felinger gave a comprehensive review of these issues [8].

The values of $P_{m}$ given by Eq. 1 are surprisingly small. They decrease exponentially with increasing square of the number of mixture components $(\mathrm{m})$ and increase exponentially with increasing peak capacity $\left(n_{c}\right)$. If the complexity of a mixture increases 10 times, the peak capacity of the column must increase 100 times to keep constant the resolution of the mixture. Analysts are resourceful and can do better than what predicts Eq. 1 and what can be achieved with a random choice of phase systems. Nevertheless, the difficulty of separating complex mixtures increases very fast with their increasing complexity.

\section{COMBINATIONS OF TWO UNIDIMENSIONAL SEPARATIONS.}

All separations result from a selective transport during which the molecules of the sample components move into different directions or at different velocities along a given direction 
[10]. The mass centers of the distributions of the molecules of the different components must be transported as far apart as possible from each other. The scatter of the molecules of a given component must be as limited as possible since it controls the resolution between compounds that moved closely. A two-dimensional separation process is effective if the distribution patterns yielded by the separations in the two directions are uncorrelated.

The separations provided by any of the known methods used in classical, unidimensional chromatography are made either in the time or in the space dimensions of the separation space. The space of separations carried out in the time dimension is a column or tube filled with a permeable bed made of packed porous particles or of a porous, permeable, monolithic solid filling the tube. Sample components are carried along this bed at velocities proportional to the solvent velocity and related to their equilibrium constants between the two phases. They are detected at the column exit. The natures of the solid material filling the column and of the solvent that percolates through it are selected to provide a sufficient separation of the sample components. Countless papers and books have been devoted to this choice. The separations made by gas chromatography (GC) or by HPLC are time separations. Their theories are now well known $[4,10]$.

Separations carried out in space use also a porous bed but, in contrast with separations made in time, the sample components are moved to different locations of this bed. At the end of the separation, the bed is scanned to locate the separated fractions. The bed used is a thin layer made of porous particles (e.g., silica, paper) coated on a solid plate [14] or placed between two rigid plates [3]. The same stationary phases can often be used in either time or space separations. The stream of mobile phase is generated by capillary forces (thin-layer chromatography or TLC) or by forcing a stream of mobile phase to percolate across the layer (over-pressurized thin layer chromatography, OPTLC). The same care must be paid to the proper selection of stationary and mobile phases as in time separations. The sample is placed in the thin layer of stationary phase, close to an edge of the plate. The liquid moves along the plate, an operation called its development.

Since unidimensional separations can be made either in a time or in a physical space, there are four possible bidimensional separations: (1) in the two perpendicular directions of a plane, giving $L C^{\times} \times L C^{x}$, known as $2 D-T L C[15]$ and still used in chemistry and biochemistry; (2) in two time dimensions, giving LC $\times$ t $\times C^{t}$ or comprehensive $2 \mathrm{D}-\mathrm{HPLC}$, implemented either on-line [2] or off-line [16-18]; (3) on a thin layer, as in $L C^{x} \times L^{x}$, followed by elution of the layer in the second direction, the wide, thin stream of eluent flowing through a detector than records the separated zones as they elute out of the wide plate. This is $L C^{x} \times L^{t}$; (4) in a column the effluent of which is nebulized, collected as a line along the side of a plate, after which the plate is developed and scanned. This is $L C^{t} \times L C^{x}$.

We studied the first three of these methods.

\section{1 - Combination of Two Time Separations.}

We developed a new separation method of peptide digests and applied it to the separation of the tryptic digests of myoglobin and bovine serum albumin (BSA). This method is based on the off-line combination of two powerful chromatographic separations [2]. It gives a 
conditional peak capacity exceeding 7000 under the best set of conditions investigated. This performance is achieved at the cost of an analysis time of the order of 28 hours.

The first separation is made on a $110 \times 4.6 \mathrm{~mm}$ column packed with Partisphere SCX, $5 \mu \mathrm{m}$ particles (Whatman, Florham Park, $\mathrm{NJ}$ ), a strong cation exchanger made of porous silica bonded to aryl-sulfonic acid chains. It was eluted with a gradient of $\mathrm{KCl}$ in a solution of $0.5 \%(\mathrm{v} / \mathrm{v})$ acetic acid in $95 / 5 \mathrm{H}_{2} \mathrm{O} /$ acetonitrile. Fractions of the effluent of this column were collected continuously with a period of $20 \mathrm{sec}$ (200 $\mu \mathrm{L}$ per fraction), giving 140 fractions. These fractions were successively injected on a column packed with $\mathrm{C}_{18}$-bonded shell silica particles (Halo, Advanced Materials Technology, Wilmington, DE), $2.7 \mu \mathrm{m}$ in diameter, which was eluted with a linear gradient of acetonitrile in water (with $0.1 \%, v / v$ TFA), from 5 to $50 \%$ acetonitrile in $4 \mathrm{~min}$, at a flow rate of $3 \mathrm{~mL} / \mathrm{min}$. Between two analysis, the column is regenerated for 4 min with a $5 \%$ aqueous solution of acetonitrile. Figure 1 shows a typical separation, as a 3-D plot of the signal intensity (in four color shades) versus the retention times on the two columns. The first separation gives a peak capacity of ca. 50 , the second a peak capacity of ca. 140, providing an overall peak capacity of 7000 [2].

Due to the fundamental differences between the molecular interactions involved between the solute and the two stationary phases used in this study, ion-exchange and reversed

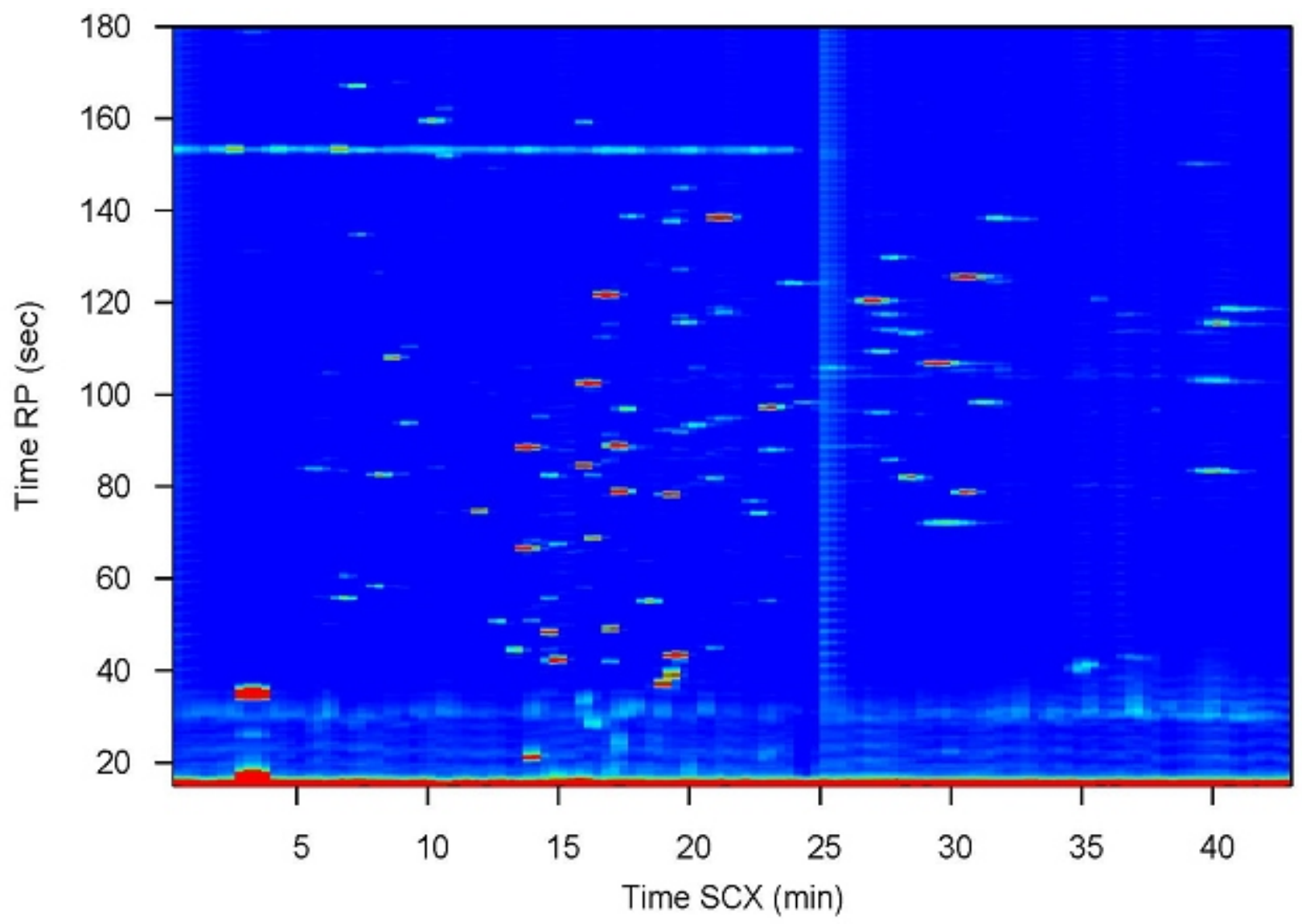

Figure 1. 2-D separations of a BSA digest, first on a cation exchanger then on a RPLC column. 
phase chromatography, the retention data of peptides on the two columns are poorly correlated, the spots corresponding to the different peptides separated are scattered over nearly the whole separation space in Fig. 1 . The differences in the analysis times on the two columns and in their efficiencies explain the differences in the two sizes of each spot.

The separation power achieved far exceeds that of any 1D separation ever published. It is an order of magnitude larger than that of most analyses published so far that combine two separations on-line [19-23]. The gain in resolution achieved is due to the selection of the very efficient Halo column for the second separation [24-26]. It gives spots that are very narrow in the second separation dimension (see Figure 2). Admittedly, this vast increase in separation power comes at the cost of a large increase in analysis time. However, a similar gain in separation power in 1D chromatography would require a nearly two orders of magnitude increase in the analysis time, making 2D chromatography a most attractive solution. The only method in which separation powers corresponding to peak capacities of a few thousands were previously achieved was Mudpit [27-30]. Our method, which is far from optimized yet, provides a peak capacity that is approximately three times larger for nearly the same analysis time as Yates' method. Compared to Mudpit, the gain is due in part to the substitution of a continuous gradient to a step gradient program for the first separation, allowing the collection of a much larger number of fractions of the first column eluent. Although less powerful than the combination of two narrow-bore columns described by Shen et al. [31] that was made possible by the use of a sophisticated mass spectrometer as the detector, our method required also a much shorter analysis time.

Figure 1 illustrates the separation power of off-line methods and the vast realm of possibilities offered to analysts by the full palette of the implementations methods of multi-dimensional liquid chromatography that are now available [1]. The main value of our work [2] is in showing that considerable improvements in the peak capacity of two-dimensional chromatography are possible, at the cost of long analysis time. Even this approach, however, is insufficient to solve many complex analytical problems. The only way to accelerate analyses is to run simultaneously all the second dimension separations, which requires the development of space-based, not time-based separation methods [1].

\section{2 - Development of an Instrument for Time-based Separations.}

We designed, constructed, and operated a pressurized, flat, wide column for high pressure liquid chromatography (HPLC). This apparatus is derived from instruments that implement over-pressured thin-layer chromatography [3]. It is designed to perform uni-dimensional LC separations but is the first step in the construction of the instrument needed to carry out two-dimensional $\mathrm{LC}^{\times} \times \mathrm{LC}^{\mathrm{t}}$ chromatographic separations. The first separation is a space-based $\mathrm{LC}^{\times}$separation taking place along one side of the flat bed. The second separation will be a time-based separation, $\mathrm{LC}^{\mathrm{t}}$, as in classical HPLC but this separation will proceed along the second dimension of the flat column, not along a tube.

Figures $2 \mathrm{a}$ and $2 \mathrm{~b}$ show a schematic of this instrument. It consists of a square column chamber (Figure 2a) and three pressurization chambers (Figure 2a), separated by a thin Mylar membrane. The $1 \mathrm{~mm}$ thick column chamber is filled with the stationary phase. 
Suitable solvent inlet and outlet ports are located on two opposite sides of the sorbent layer. The central pressurization chamber ensures effective seal of the stationary phase layer and the homogenous flow of the mobile phase percolating through the column. The entire width of the sorbent layer can be used to develop or to elute samples [3]. This instrument was designed for testing the detector needed in a two-dimensional instrument. A schematics of the detector arrangement is shown in Figure 2. The sheet of mobile phase exiting the column (left in Figure $2 \mathrm{~b}$ ) moves along a vertical rectangular Z-channel closed
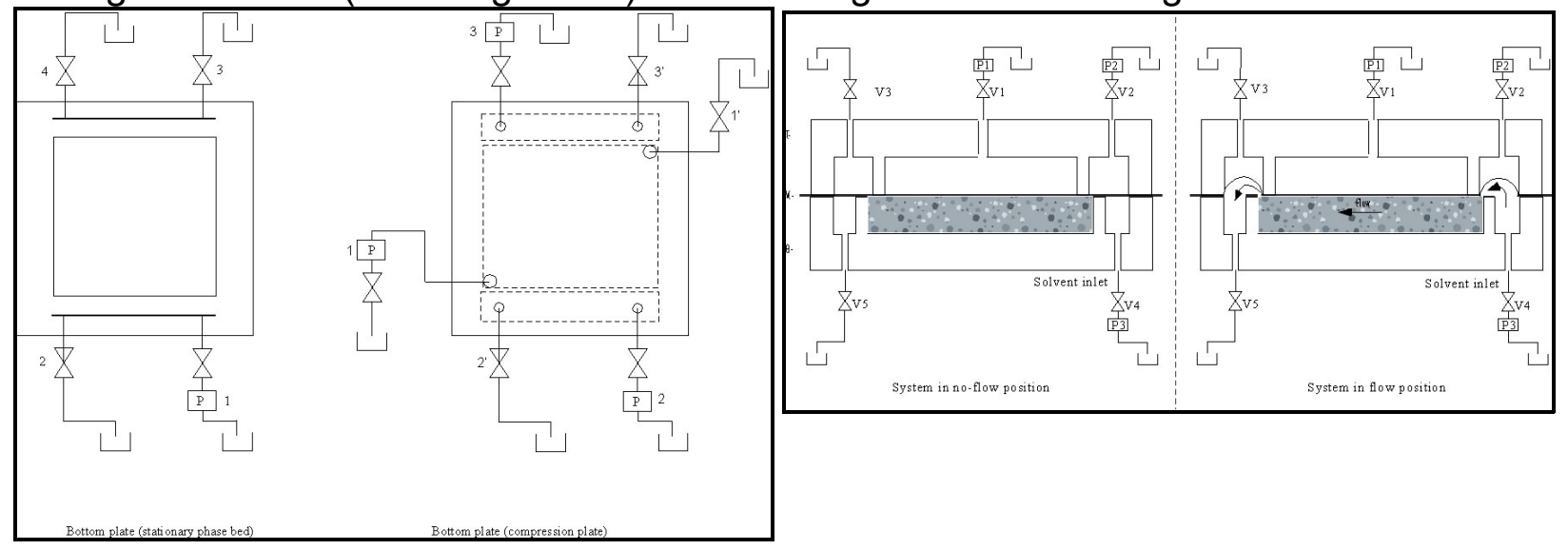

Figure 2. View of the inner faces of the top and bottom plates of the instrument. 2a-Far left: The packing material is in the central chamber. The mobile phase flows into and out of the column beds through two channel ports. $P$, mobile phase pump; $V_{i}$, needle valves. Center left: Chambers 1 and 2 serve as valves to switch on or off the mobile phase stream. $P_{i}$, pumps, $V_{i}$, needle valves for the compression fluid. $\mathbf{2} \boldsymbol{b}$-Far right: Cross-section of the instrument.

at each end with a quartz window (Fig. 3). A monochromatic UV beam passes through the channel to a diode-array detector that monitors the eluent composition in as many points as there are diodes in the array. A second detection method studied consists in placing electrochemical sensors across the exit channel and monitoring their voltage.

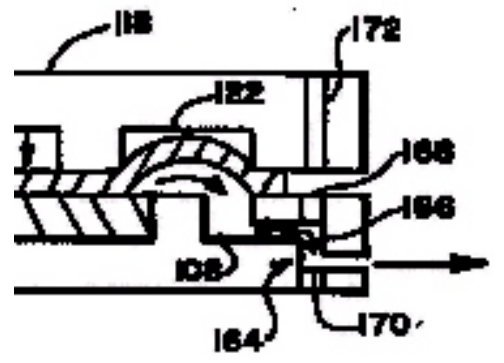

Figure 3. Schematics of the detector. 122, compression chamber; 108, solvent exit groove; 164, Z-channel; 166, detector cell; 165, 170, quartz windows; 172, open slit.

Filling the bed cavity with a thick slurry of a suitable packing material, we packed beds more radially homogeneous than columns [3]. Chromatograms exhibiting efficiencies similar to those of columns packed with the same particles were obtained. The potential limit in this efficiency comes from the physical limits of the size of the cavity and the pressure at which the instrument can be operated, markedly less than cylindrical columns can be [3].

We were not able to find a long enough diode-array with a large number of diodes to monitor the composition the whole sheet of solvent exiting the column. UV diode-array detectors used in HPLC are designed to operate as spectrometric detectors, not as chromatographic detectors. Adapting them would be too complex for us. Independent 
diode-arrays need to be monitored with dedicated electronic systems. We found a 32-diode array and built its electronic unit. In parallel, we modified an instrument similar to the one described above and adapted five micro-pH sensors to perform local detection of ionic compounds. We are using these two detector systems to measure the performance of our column. Finally, we are planning to dismantle a document scanner and use its core element to scan the optical density of the liquid stream exiting the flat column. This device has the diode density (300/in), the size, and the response speed needed. However, it is not sensitive to UV radiations. It will be used with samples made of dyes.

\section{3 - Optimization of Two-Dimensional Chromatographic Separations.}

In all implementations of two-dimensional chromatography, the separation made on the second column must be repeated a large number of times. A detailed analysis of the different components of this cycle time was made for the three possible combinations of column chromatography, on-line, stop-and-go, and off-line. This allowed a comparison of their performance in terms of peak capacity generated and total analysis time. We showed that, for a given target peak capacity, there is an optimum capacity of the first dimension column and an optimum density of the collected fractions in the first dimension. In the case of the separation of protein digests, the best performance achieved were peak capacities of 266 in 20 minutes and of 4000 in 5 hours. Experimental results confirmed these calculated performance [32-34].

Due to the time restriction of the second dimension gradient time, on-line 2D-LC schemes cannot realistically afford peak capacities exceeding 10000, even if they are implemented with exceptionally efficient columns, if long analysis times are accepted, and if several columns are used in parallel in the second dimension [34,35].

One of the most serious drawbacks of 2D-LC is the dilution of analytes during their migration through the two columns. We developed an equation providing the dilution factors as a function of the experimental conditions. The net dilution factor is between 200 and 300 in typical 2D-LC separations. Our results show that the optimization of the experimental conditions must be made from the points of view of both the retention factor and the detector response factor [36]. 


\section{REFERENCES CITED}

[1] Implementations of Two-dimensional Liquid Chromatography. G. Guiochon, N. Marchetti, K. S. Mriziq, R. A. Shalliker. J. Chromatogr. A, 2008, 1189, 109-168.

[2] Comprehensive Off-line Bi-Dimensional Liquid Chromatographic. Separations of Peptides by a Combination of two Gradient Elutions, one by a Strong Cation Exchange column, the other by Reversed Phase. N. Marchetti, J. N. Fairchild G. Guiochon. Anal. Chem., 2008, 80, 2756-2767.

[3] Column Properties and Flow Profiles in Over-pressured Thin Layer Chromatography. K. S. Mriziq, G. Guiochon. J. Chromatogr. A, 2007, 1187, 180-187.

[4] Ultimate Limit of the Separation Power in Unidimensional Column Liquid Chromatography. G. Guiochon. J. Chromatogr. A, 2006, 1126, 6-49.

[5] Maximum number of components resolvable by gel filtration and other elution chromatographic methods, J. C. Giddings, Anal. Chem., 1967, 39, 1027.

[6] Chromatographic peak capacity and the factors influencing it, E. Grushka, Anal. Chem., 1970, 42, 1142.

[7] Theoretical Investigation of the Optimum Particle Size for the Resolution of Proteins by Size Exclusion Chromatography, M. Martin and G. Guiochon, J. Chromatogr., 1985, 326, 3.

[8] Statistical Method for Estimation of Number of Components from Single Complex Chromatograms: theory, Computer-Based Testing, an Analysis of Errors, J. M. Davis and J. C. Giddings, Anal. Chem., 1985, 57, 2168.

[9] Data Analysis and Signal Processing in Chromatography, A. Felinger, Elsevier, Amsterdam, The Netherlands, 1998.

[10] Unified Separation Science, J. C. Giddings, Wiley, New York, NY, 1991.

[11] Influence of the peak height distribution on separation performances - discrimination factor and effective peak capacity, M. Z. El Fallah, M. Martin, Chromatographia, $1987,24,115-122$.

[12] On the potential of 2-dimensional and multidimensional separation systems, $\mathrm{M}$. Martin, Fresenius J. Anal. Chem., 1995, 352, 625-632. 
[13] Probability Distributions of the Number of Chromatographically Resolved Peaks and Resolvable Components in Mixtures, M. Martin, D. P. Herman and G. Guiochon, Anal. Chem., 1986, 58, 2200.

[14] Fundamentals of Thin-Layer Chromatography. (Planar Chromatography), F. Geiss, Hüthig Verlag, Heidelberg, Germany, 1987.

[15] Qualitative analysis of proteins: a partition chromatography method using paper, $R$. Consden, A. H. Gordon and A. J. P. Martin, Biochem. J., 1944, 38, 224.

[16] D. R. Stoll, X. Li, X. Wang, P. W. Carr, Sarah E.G. Porter, Sarah C. Rutan, J. Chromatogr. A, 2007, 1168, 3-43.

[17] P. J. Schoenmakers, G. Vivó-Truyols, W. M.C. Decrop, J. Chromatogr. A, 2006, 1120, 282-290.

[18] P. Jandera, P. Česla, T. Hájek, G. Vohralík, K. Vyňuchalová, J. Fischer, J. Chromatogr. A, 2007, 1189, 207-220.

[19] X. Jiang, A. van der Horst, V. Lima, P. J. Schoenmakers, J. Chromatogr. A, 2005, 1076, 51.

[20] D. R. Stoll, P. W. Carr, J. Am. Chem. Soc., 2005, 127, 5034.

[21] D. R. Stoll, J. D. Cohen, P. W. Carr, J. Chromatogr. A, 2006, 1122, 123

[22] P. Dugo, T. Kumm, M. L. Crupi, A. Cotroneo, L. Mondello, J. Chromatogr. A, 2006, 1112, 269.

[23] P. Dugo, V. \{lu S\}ke\{lu r\}ikov\{l'a\}, T. Kumm, A. Trozzi , P. Jandera, L. Mondello, Anal. Chem., 2006, 78, 7743.

[24] N. Marchetti, A. Cavazzini, F. Gritti, G. Guiochon, J. Chromatogr. A, 2007, 1163, 203.

[25] A. Cavazzini, F. Gritti, K. Kaczmarski, N. Marchetti and G. Guiochon, Anal. Chem., 2007, 79, 5972.

[26] F. Gritti, A. Cavazzini, N. Marchetti, G. Guiochon, J. Chromatogr. A, 2007, 1157, 289.

[27] Direct analysis of protein complexes using mass spectrometry, A. J. Link, J. Eng, D. M. Schieltz, E. Carmack, G. J. Mize, D. R. Morris, B. M. Garvick, and J. R. Yates 
III, Nature Biotechnol., 1999, 17, 676-682.

[28] Large-scale analysis of the yeast proteome by multidimensional protein identification technology, M. P. Washburn, D. Wolters and J. R. Yates III, Nature Biotechnol., 2001, 19, 242-247.

[29] An automated multidimensional protein identification technology for shotgun proteomics, D. Wolters, M. P. Washburn and J. R. Yates III, Anal. Chem., 2001, 73, 5683-5690.

[30] Analysis of quantitative proteomic data generated via multidimensional protein identification technology, M. P. Washburn, R. Ulaszek, C. Deciu, D. M. Schieltz and J. R. Yates III, Anal. Chem., 2002, 74, 1650-1657.

[31] Y. Shen, J. M. Jacobs, D. G. Camp II, R. Fang, R. J. Moore, R. D. Smith, W. Xiao, R. W. Davis, R. G. Tompkins, Anal. Chem., 2004, 76, 1134.

[32] K. Horvàth, J. N. Fairchild, G. Guiochon, J. Chromatogr. A, 2009, 1216, 2511-2518.

[33] J. N. Fairchild, K. Horváth, G. Guiochon, J. Chromatogr. A, 2009, 1216, 1363-1371.

[34] K. Horváth, J. N. Fairchild, G. Guiochon. Anal. Chem., 2009, 81, 3879-3888.

[35] J. N. Fairchild, K. Horvath, G. Guiochon. J. Chromatogr. A, 2009, 1216, 6210-6217.

[36] K. Horváth, J. N. Fairchild, G. Guiochon. J, Chromatogr. A, 2009, 1216, 7785-7792. 


\section{ANNEX}

\section{LIST of 2007-2009 PUBLICATIONS SUPPORTED by GRANT DE-FG05-88ER13859}

Publications of results obtained with the support of previous efforts made under different titles, with different emphasis are still being published.

\section{Homogeneity of an Adsorbent Surface.}

(1) Thermodynamics of adsorption of binary aqueous organic liquid mixtures on a RP-HPLC adsorbent. F. Gritti, G. Guiochon. J. Chromatogr. A, 2007, 1155, 85-99.

(2) Experimental evidence of the influence of the surface chemistry of the packing material on the column pressure drop in RPLC. F. Gritti, G. Guiochon. J. Chromatogr. A, 2006, 1136, 192-201.

(3) Measurement of Hold-up Volumes in RP-HPLC. Definition and comparison between static and dynamic methods. F. Gritti, Y. V. Kazakevich, G. Guiochon. J. Chromatogr. A, 2007, 1161, 157-169.

(4) Effect of the surface coverage of endcapped C18-silica on the excess adsorption isotherms of commonly used organic solvents from water in RPLC. F. Gritti, Y. V. Kazakevich, G. Guiochon. J. Chromatogr. A, 2007, 1169, 111-124.

(8) Elution Band profiles of acids and bases in reversed-phase liquid chromatography. F. Gritti, G. Guiochon. J. Sep. Sci., 2008, 31, 3657-3682.

(9) Peak shapes of acids and bases underoverloaded conditions in Reversed Phase Liquid Chromatography, with mobile phases of various buffer $\mathrm{pH}$. A thermodynamic interpretation. F. Gritti, G. Guiochon. J. Chromatogr. A, 2009, 1216, 63-78.

(10) Adsorption mechanism of acids and bases in RPLC in weak buffered mobile phases designed for LC/MS. F. Gritti, G. Guiochon. J. Chromatogr. A, 2009, 1216, 1776-1788.

(11) Determination of the adsorption energy distribution of neutral and charged compounds onto endcapped silica- $C_{18}$ adsorbents from polar liquid phases. Fabrice Gritti and Georges Guiochon.Colloid Journal (Kolloidnyi zhurnal), 2009, 71(4), 479-485.

(12) Adsorption mechanisms in hydrophilic interaction chromatography and in per aqueous liquid chromatography. Fabrice Gritti, Alberto dos Santos Pereira, Pat Sandra and Georges Guiochon. Journal of Chromatography A, 2009,1216, 8496-8504. 


\section{Study of The Homogeneity of a Chromatographic Column}

(13) Prediction/Verification of Particle Motion in One Dimension with the Discrete Element Method. Feng Chen, Eric C. Drumm, Georges Guiochon. ASCE International J. Geomechanics, 2007, 7, 344-352.

NB. This paper received a "2008 Best Paper Award" from the association (IACMAG) that publishes the journal.

(14) Structural Radial Heterogeneity of a Silica-Based Wide-Bore Monolithic Column. Khaled S. Mriziq, Jude A. Abia, Youngmi Lee, and Georges Guiochon. J. Chromatogr. A. 2008,1195, 97-103.

(15) Discrete Element Simulation of 1D Upward Seepage Flow with Particle-Fluid Interaction Using Coupled Open Source Software, F. Chen, E. C., Drumm, G. Guiochon, and K. Suzuki, Paper \# 730098903 Proceedings of The 12th International Conference of the International Association for Computer Methods and Advances in Geomechanics (IACMAG), Goa, India , 1-6 October, 2008.

\section{Separation of Highly Complex Mixtures by Two-dimension Liquid Chromatography}

(16) Column Properties and Flow Profiles in Over-pressured Thin Layer Chromatography. K. S. Mriziq, G. Guiochon. J. Chromatogr. A, 2007, 1187, 180-187.

(17) Implementations of Two-dimensional Liquid Chromatography. G. Guiochon, N. Marchetti, K. Mriziq, R. A. Shalliker. J. Chromatogr. A, 2008, 1189, 109-168.

(18) Comprehensive Off-line Bi-Dimensional Liquid Chromatographic. Separations of Peptides by a Combination of two Gradient Elutions, one by a Strong Cation Exchange column, the other by Reversed Phase. N. Marchetti, J. N. Fairchild, G. Guiochon. Anal, Chem., 2008, 80, 2756-2767.

(19) Optimization strategies for off-line two-dimensional liquid chromatography. Krisztían Horvàth, Jacob Fairchild, Georges Guiochon, J. Chromatogr. A, 2009, 1216, 2511-2518.

(20) Approaches to Comprehensive Two-Dimensional Liquid Chromatography Systems. Jacob N. Fairchild, Krisztián Horváth, Georges Guiochon, J. Chromatogr. A, 2009, 1216, 1363-1371.

(21) Optimization of on-line two-dimensional chromatography. Krisztián Horváth, Jacob Fairchild, Georges Guiochon. Anal. Chem., 2009, 81, 3879-3888.

(22) Theoretical advantages and drawbacks of on-line, multidimensional liquid 
chromatography using multiple columns operated in parallel. Jacob N. Fairchild, Krisztian Horvath, Georges Guiochon. J. Chromatogr. A, 2009, 1216, 6210-6217.

(23) Detection issues in two-dimensional liquid chromatography. Krisztil'an Horvl'ath, Jacob N. Fairchild, Georges Guiochon. J, Chromatogr. A, 2009, 1216, 7785-7792. 